\title{
Análisis de la sección de discusión de los artículos científicos de los docentes investigadores de la UNCP, 2008-2012
}

\section{Analysis of the discussion section of the scientific articles of the faculty researchers UNCP, 2008-2012}

\author{
Miguel Miguel,Hugo' / Hurtado Rivera, Jesús² \\ 'hmiguel21@gmail.com \\ 2jahr38@hotmail.com \\ Facultad de Ciencias Forestales y del Ambiente de la Universidad Nacional del Centro del Perú
}

\section{RESUMEN}

Se analizó la discusión de los artículos científicos elaborados por los docentes investigadores de la UNCP, entre los años 2008 al 2012. La población del estudio estuvo constituida por todos los artículos científicos producidos anualmente por los docentes investigadores. La muestra de estuvo compuesta por 48 artículos, obtenidos probabilísticamente; para $\mathrm{N}=2118 ; \mathrm{Z}_{\square}{ }^{2}=$ 1,962 ; para una seguridad del $95 \%$; $p=$ proporción esperada $(5 \%=0.05) ; q=1-p$ $(1-0.05=0.95) ; d=$ Precisión $(10 \%)$. En cada facultad se eligieron al azar 6 artículos científicos. Los datos del estudio provienen de la base de datos de los investigadores del Centro de Investigación de la UNCP. Con los hallazgos conseguidos se concluye que; los porcentajes de los niveles de la interpretación de los datos son: $39.6 \%$ de nivel mediano; $52.1 \%$ de nivel bajo; y $8.3 \%$ de nivel nulo; los porcentajes de los niveles de implicaciones de los datos son: $6.3 \%$ de nivel bajo; y $93.8 \%$ de nivel nulo; los porcentajes de los niveles de definición de las potenciales limitaciones del estudio son: $6.3 \%$ del nivel mediano; el 4.2 $\%$ del nivel bajo; y $89.6 \%$ de nivel nulo; los porcentajes de los niveles de concordancias o discordancias con publicaciones anteriores son: $12.5 \%$ de nivel mediano; $20.8 \%$ de nivel bajo; y $66.7 \%$ de nivel nulo; y los

\section{ABSTRACT}

The discussion of scientific articles written by researchers of the UNCP teachers, between 2008 to 2012 was analyzed. The study population consisted of all scientific papers produced annually by educational researchers. The sample consisted of 48 items, obtained probabilistically; for $\mathrm{N}=2118$; Z $\square 2=1,962$; for a $95 \%$ confidence; $p=$ expected proportion $(5 \%=0.05) ; p q=1-(1-.05=0.95) ; d=$ Accuracy (10\%). In each school they were randomly selected 8 scientific papers. The survey data come from the database of the researchers of the Research Center of the UNCP. With the achieved findings we conclude that; the percentages of levels of data interpretation are: $39.6 \%$ medium level; $52.1 \%$ low level; and $8.3 \%$ from zero level; percentages levels implications of the data are: $6.3 \%$ low level; and $93.8 \%$ of zero level; the percentages of levels of definition of the potential limitations of the study are: $6.3 \%$ of the median level; $4.2 \%$ of the low level; and $89.6 \%$ of zero level; the percentages of the levels of concordance or discordance with previous publications include: $12.5 \%$ of medium level; $20.8 \%$ low level; and $66.7 \%$ of zero level; and the percentages of the levels of the practical applications of the results are: $6.3 \% \%$ low level; $93.8 \%$ of zero level. 
porcentajes de los niveles de las aplicaciones prácticas de los resultados son: $6.3 \%$ de nivel bajo; $93.8 \%$ de nivel nulo.

Palabras clave: Artículo científico, discusión, investigación.
Keywords: Journal article, discussion, research.

\section{INTRODUCCIÓN}

La discusión es más dificultoso de precisar que las demás secciones de un artículo científico; por ello, es también, normalmente, la sección más difícil de escribir; numerosos artículos son rechazados por los directores de revistas por una discusión deficiente, aunque los datos del documento sean válidos e interesantes, (1). La discusión de los datos en los artículos científicos corresponde a la forma como los resultados son interpretados por el investigador, tanto en función de la hipótesis planteada, como a la de lo que otros autores expresan o han encontrado sobre el tema.

Se trata de explicar qué significan los resultados y por qué ocurrieron de ese modo las cosas, 0 sea la interpretación de los datos en base a la totalidad de la evidencia disponible, incluidas las fuentes de sesgo e imprecisión (validez interna) y la discusión de la validez externa, incluyendo las medidas cuantitativas cuando sea posible, (2).

En consecuencia, con lo expuesto, existe la necesidad de estudiar el desarrollo de la sección de la discusión en la redacción de los artículos científicos que elaboran los docentes de la UNCP, por lo que se diseñó el siguiente objetivo: analizar la sección de la discusión de los artículos científicos producidos por los docentes investigadores de la UNCP, durante los años 2008 al 2012.

\section{MATERIAL Y MÉTODOS}

\section{Método de Investigación}

La investigación fue del tipo aplicado, de ni- vel descriptivo transversal, por lo que se buscó analizar la discusión de los artículos científicos elaborados por los docentes investigadores de la UNCP, entre los años 2008 al 2012. (Hernández, Cazau), (5 y 6).

El nivel de investigación fue descriptivo, porque exhibe el conocimiento de la realidad tal como se presenta en una situación de espacio y de tiempo dado. Se observa y se registra, o se pregunta y se registra. Describe el fenómeno sin introducir modificaciones, (7).

La población del estudio estuvo constituida por todos los artículos científicos producidos anualmente por los docentes investigadores de la UNCP, durante 5 años. La muestra fue probabilística, representada por el $5 \%$ de la población de los artículos científicos, distribuidos en las 24 facultades; de los cuales se muestrearon las siguientes: Agronomía, Ciencias Forestales y del Ambiente; Medicina Humana; Ingeniería Química; Sociología; Ciencias Agrarias (Satipo); Economía; y Arquitectura. La muestra de artículos científicos estuvo compuesta por 48 artículos, obtenidos probabilísticamente; para $\mathrm{N}$ $=2027 ; Z_{\square}^{2}=1,962$; para una seguridad del $95 \% ; p=$ proporción esperada $(5 \%=0.05) ; q$ $=1-\mathrm{p}(1-0.05=0.95) ; \mathrm{d}=$ Precisión $(10 \%)$. En cada facultad se eligieron al azar 6 artículos científicos.

Los datos del estudio provienen de los archivos del Centro de Investigación de la UNCP. Los materiales y equipos que se utilizaron: Bases de datos del Centro de Investigación de la UNCP, fichas de evaluación, y equipo informático.

\section{Procedimiento:}

Una vez ubicados al azar los artículos científicos, 
se les aplicó el formato de evaluación por niveles (alto, mediano, bajo y nulo), de los ítems: interpretación de los resultados; implicaciones de los resultados; potenciales limitaciones del estudio; concordancias o discordancias con pu- blicaciones anteriores; y aplicaciones prácticas de los resultados, siguiendo la secuencia lógica de la matriz de operacionalización de las variables.

Tabla 1. Matriz de Operacionalización de las variables:

\begin{tabular}{|c|c|c|c|c|}
\hline Variables & Indicadores & Unidad & Instrumento & Fuente \\
\hline \multirow{5}{*}{ Discusión } & Interpretación de los resultados & \multirow{5}{*}{$\begin{array}{l}\text { Niveles: } \\
\text { (porcentajes) } \\
\text { Alto, mediano, } \\
\text { bajo y nulo }\end{array}$} & Ficha de evaluación & $\begin{array}{l}\text { Archivos de } \\
\text { CIUNCP }\end{array}$ \\
\hline & Implicaciones de los resultados & & Ficha de evaluación & $\begin{array}{l}\text { Archivos de } \\
\text { CIUNCP }\end{array}$ \\
\hline & & & Ficha de evaluación & $\begin{array}{l}\text { Archivos de } \\
\text { CIUNCP }\end{array}$ \\
\hline & $\begin{array}{l}\text { Concordancias o discordancias con } \\
\text { publicaciones anteriores }\end{array}$ & & Ficha de evaluación & $\begin{array}{l}\text { Archivos de } \\
\text { CIUNCP }\end{array}$ \\
\hline & $\begin{array}{l}\text { Aplicaciones prácticas de los } \\
\text { resultados }\end{array}$ & & Ficha de evaluación & $\begin{array}{l}\text { Archivos de } \\
\text { CIUNCP }\end{array}$ \\
\hline
\end{tabular}

Para el contraste de la hipótesis: "Los indicadores de la calidad de discusión de los artículos científicos producidos por los docentes inves- tigadores de la UNCP son bajos", se utilizó la escala de la propuesta de un modelo de evaluación de tesis doctorales.

$\begin{array}{cccc}\text { Nulo (1) } & \text { Bajo (2) } & \text { Mediano (3) } & \text { Alto (4) } \\ 0 \text { a } 25 \% & 25.1 \text { a } 50 \% & 50.1 \text { a } 75 \% & 75.1 \text { a } 100 \%\end{array}$

\section{RESULTADOS}

Evaluación del nivel de la interpretación de los resultados, en la discusión de los artículos científicos

Tabla 2. Porcentajes de los niveles (alto, mediano, bajo y nulo) de la interpretación de los resultados en la discusión de los artículos científicos.

\begin{tabular}{llcccc}
\hline & \multicolumn{1}{c}{ Facultad } & \multicolumn{4}{c}{ Nivel de Interpretación de los Datos } \\
\cline { 3 - 6 } & Alto & Mediano & Bajo & Nulo \\
\hline 1. & Agronomía & 0 & 5 & 1 & 0 \\
2. Ciencias Forestales y del Amb. & 0 & 2 & 4 & 0 \\
3. Medicina Humana & 0 & 3 & 2 & 1 \\
4. Ingeniería Química & 0 & 1 & 4 & 1 \\
5. Sociología & 0 & 0 & 6 & 0 \\
6. Ciencias Agrarias Satipo & 0 & 3 & 2 & 1 \\
7. & Economía & 0 & 3 & 3 & 0 \\
8. & Arquitectura & 0 & 2 & 3 & 1 \\
\hline & Suma: & 0 & 19 & 25 & 4 \\
& Promedio: & 0 & 2.4 & 3.1 & 0.5 \\
$\quad$ \% & 0.0 & 39.6 & 52.1 & 8.3 \\
\hline
\end{tabular}


Evaluación del nivel de inclusión de las implicaciones de los resultados en la discusión de los artículos científicos

Tabla 3. Porcentajes de los niveles (alto, mediano, bajo y nulo) de implicaciones de los datos en la discusión de los artículos científicos.

\begin{tabular}{llcccc}
\hline & \multirow{2}{*}{ Facultad } & \multicolumn{4}{c}{ Nivel de Implicaciones de los Datos } \\
\cline { 3 - 6 } & Alto & Mediano & Bajo & Nulo \\
\hline 1. & Agronomía & 0 & 0 & 0 & 6 \\
2. $\quad$ Ciencias Forestales y del Amb. & 0 & 0 & 1 & 5 \\
3. Medicina Humana & 0 & 0 & 2 & 4 \\
4. Ingeniería Química & 0 & 0 & 0 & 6 \\
5. Sociología & 0 & 0 & 0 & 6 \\
6. & Ciencias Agrarias Satipo & 0 & 0 & 0 & 6 \\
7. $\quad$ Economía & 0 & 0 & 0 & 6 \\
8. & Arquitectura & 0 & 0 & 0 & 6 \\
\hline & Suma: & 0 & 0 & 3 & 45 \\
& Promedio: & 0 & 0.0 & 0.4 & 5.6 \\
$\quad \%$ & 0.0 & 0.0 & 6.3 & 93.8 \\
\hline
\end{tabular}

Evaluación de la definición de las potenciales limitaciones del estudio en la discusión de los artículos científicos

Tabla 4. Porcentajes de los niveles (alto, mediano, bajo y nulo) de la definición de las potenciales limitaciones del estudio en la discusión de los artículos científicos.

\begin{tabular}{|c|c|c|c|c|}
\hline \multirow{2}{*}{ Facultad } & \multicolumn{4}{|c|}{$\begin{array}{c}\begin{array}{c}\text { Nivel de definición de las potenciales limitaciones del } \\
\text { estudio }\end{array} \\
\end{array}$} \\
\hline & Alto & Mediano & Bajo & Nulo \\
\hline 1. Agronomía & 0 & 0 & 0 & 6 \\
\hline 2. Ciencias Forestales y del Amb. & 0 & 0 & 0 & 6 \\
\hline 3. Medicina Humana & 0 & 0 & 1 & 5 \\
\hline 4. Ingeniería Química & 0 & 0 & 0 & 6 \\
\hline 5. Sociología & 0 & 0 & 0 & 6 \\
\hline 6. Ciencias Agrarias Satipo & 0 & 0 & 0 & 6 \\
\hline 7. Economía & 0 & 1 & 0 & 5 \\
\hline 8. Arquitectura & 0 & 2 & 1 & 3 \\
\hline Suma: & 0 & 3 & 2 & 43 \\
\hline Promedio: & 0 & 0.4 & 0.3 & 5.4 \\
\hline$\%$ & 0.0 & 6.3 & 4.2 & 89.6 \\
\hline
\end{tabular}


Evaluación de las concordancias o discordancias con publicaciones anteriores en la discusión de los artículos científicos

Tabla 5. Porcentajes de los niveles (alto, mediano, bajo y nulo) de las concordancias o discordancias con publicaciones anteriores en la discusión de los artículos científicos.

\begin{tabular}{llcccc}
\hline \multirow{2}{*}{ Facultad } & \multicolumn{4}{c}{ Nivel de las concordancias o discordancias con } \\
& & Alto & Mediano & Bajo & Nulo \\
\cline { 2 - 5 } & & 0 & 0 & 1 & 5 \\
1. Agronomía & 0 & 2 & 2 & 2 \\
2. Ciencias Forestales y del Amb. & 0 & 3 & 1 & 2 \\
3. Medicina Humana & 0 & 0 & 1 & 5 \\
4. Ingeniería Química & 0 & 0 & 1 & 5 \\
5. Sociología & 0 & 1 & 4 & 1 \\
6. Ciencias Agrarias Satipo & 0 & 0 & 0 & 6 \\
7. Economía & 0 & 0 & 0 & 6 \\
8. Arquitectura & 0 & 6 & 10 & 32 \\
\hline & Suma: & 0 & 0.8 & 1.3 & 4.0 \\
& Promedio: & 0.0 & 12.5 & 20.8 & 66.7 \\
\hline
\end{tabular}

Evaluación de las aplicaciones prácticas de los resultados en la discusión de los artículos científicos.

Tabla 6. Porcentajes de los niveles (alto, mediano, bajo y nulo) de aplicaciones prácticas de los resultados en la discusión de los artículos científicos.

\begin{tabular}{llcccc}
\hline \multirow{2}{*}{ Facultad } & \multicolumn{4}{c}{ Nivel de aplicaciones prácticas de los resultados } \\
\cline { 3 - 6 } & Alto & Mediano & Bajo & Nulo \\
\hline 1. & Agronomía & 0 & 0 & 0 & 6 \\
2. & Ciencias Forestales y del Amb. & 0 & 0 & 1 & 5 \\
3. & Medicina Humana & 0 & 0 & 2 & 4 \\
4. Ingeniería Química & 0 & 0 & 0 & 6 \\
5. & Sociología & 0 & 0 & 0 & 6 \\
6. & Ciencias Agrarias Satipo & 0 & 0 & 0 & 6 \\
7. & Economía & 0 & 0 & 0 & 6 \\
8. & Arquitectura & 0 & 0 & 0 & 6 \\
\hline & Suma: & 0 & 0 & 3 & 45 \\
& Promedio: & 0 & 0.0 & 0.4 & 5.6 \\
& $\%$ & 0.0 & 0.0 & 6.3 & 93.8 \\
\hline
\end{tabular}




\section{Análisis de la sección de la discusión de los artículos científicos producidos por los docentes investigadores de la UNCP, durante los años 2008 al 2012}

Tabla 7. Evaluación del desarrollo de la sección de la discusión de los artículos científicos producidos por docentes investigadores de la UNCP, durante los años 2008 al 2012.

Niveles de Aplicación (en porcentajes, del promedio a escala de la UNCP)

\begin{tabular}{lcccc} 
& Alto & Mediano & Bajo & Nulo \\
\hline & 0.0 & 39.6 & 52.1 & 8.3 \\
1. Interpretación de los Datos & 0.0 & 0.0 & 6.3 & 93.8 \\
2. Implicaciones de los Datos & 0.0 & 6.3 & 4.2 & 89.6 \\
3. Definición de las potenciales limitaciones del estudio & & & & \\
$\quad$ Concordancias o discordancias con publicaciones & & 12.5 & 20.8 & 66.7 \\
4. anteriores & 0.0 & 0.0 & 0.4 & 5.6 \\
5. Aplicaciones prácticas de los resultados & 0 & & & \\
\hline
\end{tabular}

\section{DISCUSIÓN}

El nivel de interpretación de los datos obtenidos en la investigación científica de los docentes de la Universidad Nacional del Centro de Perú, se observa en el cuadro 1, en el cual, el $39.6 \%$ es mediano, el $52.1 \%$ es bajo y el $8.3 \%$ es nulo; estas cifras que van desde el nivel mediano a nulo, significa que los docentes no interpretan adecuadamente sus resultados en relación a los objetivos originales e hipótesis y al estado de conocimiento actual del tema en estudio, tal como recomienda Villarán, (3).

El nivel de inclusión de las implicaciones de los resultados de la investigación científica de los docentes de la Universidad Nacional del Centro de Perú, se muestran en la tabla 2, en donde el $6.3 \%$ es bajo, y el $93.8 \%$ es nulo; estas cifras que van desde el nivel bajo a nulo; esto es muy preocupante; lo que quiere decir, que los docentes no indican la importancia o los alcances de sus hallazgos, no hacen sugerencias acerca del modo en que sus resultados podrían emplearse para mejorar las prácticas de las ciencias y pueden hacer recomendaciones encaminadas a incrementar el conocimiento en ese campo específico profundizando en las investigaciones, como sugiere Day (4).

El nivel de la definición de las potenciales limitaciones del estudio en la investigación científica de los docentes de la Universidad Nacional del Centro de Perú, se muestran en la tabla 3 , en donde el $6.3 \%$ es mediano, el $4.2 \%$ es bajo y el $89.6 \%$ es nulo; estas cifras que van desde el nivel mediano a nulo, significa que la mayoría de los docentes no indican las potenciales limitaciones del estudio; no obstante que el investigador se encuentra en la mejor posición para plantear las limitaciones del estudio, como deficiencias de la muestra, problemas de diseño, dificultades en la colecta de datos, etc. El hecho de que en la discusión se presenten estas limitaciones demuestra a los lectores que el autor estaba consciente de ellas y que quizá las tomó en cuenta al interpretar los resultados, como propone Day (4).

El nivel de las concordancias o discordancias con publicaciones anteriores en la sección de la discusión de los artículos científicos de los docentes de la Universidad Nacional del Centro de Perú, se muestran en la tabla 4 , en donde el $12.5 \%$ es mediano, el $20.8 \%$ es bajo y el $66.7 \%$ es nulo; estas cifras que van desde el nivel mediano a nulo; esto se interpreta, que la mayoría de los docentes no indican las concordancias o discrepancias con publicaciones anteriores; no obstante que la literatura existente, revela que la discusión debe ser clara y consistente con los resultados, sus características más relevantes se centran en mostrar las relaciones y generalizaciones según los cuadros, se deben 
señalar las excepciones o faltas de correlación y concretar los aspectos no resueltos sin alterar los datos, señalar las concordancias o discordancias con las publicaciones anteriores, enunciar las aplicaciones prácticas de los resultados, formular las conclusiones con claridad y resumir las pruebas que respalden cada conclusión, como recomienda, Day (4).

Visualizando en forma global los resultados (tabla 6), la evaluación del desarrollo de la sección de la discusión de los artículos científicos producidos por docentes investigadores de la UNCP, durante los años 2008 al 2012 se ubican mayoritariamente en los niveles bajo y nulo, las implicaciones de los datos, las definiciones de las potenciales limitaciones del estudio, y las aplicaciones prácticas de los resultados; el criterio de la interpretación de los datos y las concordancias o discordancias con publicaciones tienen porcentajes más o menos rescatables ubicados en los niveles medianos; estos hallazgos probablemente esté relacionados con la baja calidad del manejo del marco teórico por parte de los docentes investigadores.

\section{REFERENCIAS BIBLIOGRÁFICAS}

1. Moyeta D. (2009). La sección discusión del artículo de investigación científica en Psicología. Universidad Católica de Córdova. Argentina.

2. Polit DF, Hungler BP. (2011). Investigación científica en ciencias de la salud. Sexta edición. México: Editorial Interamericana; 2000. Citado por: Eslava J, Alzate JP. Cómo elaborar la discusión de un artículo científico. Rev. Col. Or. Tra. Colombia. 25 (1): 14-7.

3. Villagrán A, Harris PR. (2009). Algunas claves para escribir correctamente un artículo científico. Rev Chil Pediatr; 80 (1): 70 -78.

4. Day R. (2005). Cómo escribir y publicar trabajos científicos. Tercera edición en español. OPS. Washington.

5. Hernández R, Fernández C, Baptista $P$. (1999). Metodología de la investigación. Segunda Edición. Mc GRAW - HILL. México.

6. Cazau P. (2006). Introducción a la investigación en ciencias sociales. Tercera Edición. Buenos Aires.
7. Rojas M. (2002). Manual de investigación y redacción científica. Book xpress. San Isidro. Lima, Perú.

8. De Miguel M. (2010). La evaluación de tesis doctorales. Propuesta de un modelo. Relieve, Vol. 16, No 1, pp. 1-18. 